\title{
System for receiving and processing diversity acoustic noise control data
}

\author{
$V . V$. Tersin $^{1}, V . V$. Bulkin $^{2,}$, and T. D. Khromulina ${ }^{3}$ \\ ${ }^{1}$ Murom Plant of Radio Measuring Instruments, 2, Karacharovskoye shosse str., Murom, 602265, \\ Russia \\ ${ }^{2}$ Vladimir State University named after Alexader Grigoryevich and Nickolay Grigoryevich Stoletovs, \\ Murom Institute, 23, Orlovskaya str., Murom, 602264, Russia \\ ${ }^{3}$ Vladimirteplogaz, Murom Branch, 110-a, Pervomayskaya str., Murom, 602263, Russia
}

\begin{abstract}
The results of the development and experimental verification of a system for obtaining and processing diversity acoustic noise control data are presented. The system is implemented using the LabView $\mathrm{G}$ graphical programming language. The length of the measured realization is determined by the ratio of the sample size to the sampling frequency. The number of measurements of each sound signal is determined by the ratio of the analysis time to the length of the measured realization. The measured audio signal implementation in the form of 40,000 16-bit binary numbers is processed using the fast Fourier transform. The result is a discrete spectrum of the audio signal in the range from 0 to $20 \mathrm{KHz}$ with a step of 1 $\mathrm{Hz}$. The results of the system check using a laboratory acoustic chamber and a noise shield are presented. The advantages and disadvantages of the system are formulated.
\end{abstract}

\section{Introduction}

The noise problem in urban areas and in production area as well as residential premises is becoming one of the most significant technosphere problems. Acoustic noise, occupying the third place among environmental hazards, has a significant adverse effect on the psychological and biological health of a person, the state of the social environment [1]. At the same time, the noise level in the technosphere increases annually by no less than $0.5 \mathrm{~dB}$ per year, which makes the task of studying and analyzing acoustic noise of technogenic origin one of the most relevant [2].

At present, sound level meters used for noise control belong to different classes and have different capabilities [3]. In the classic version, the sound level meter is a measuring system in which the microphone performs the function of the sensor, there is an amplifier, a set of band-pass filters, and the result is displayed either using a dial gauge or a digital display. Evaluation is carried out either on average over the entire range, or at the geometric mean frequencies of the octave ranges. More complex systems provide simultaneous measurements in the octave (pre-octave) ranges, making it possible to save the measurement results, to carry out built-in and computer processing of the received information.

* Corresponding author: vvbulkin@mail.ru 
Specialized systems allow us to evaluate additional parameters, perform specialized calculations. For example, in [4] a system for ecological and meteorological monitoring of the environment is designed. The main advantage of the system is the use of fast Fourier transform (FFT) to obtain a full spectrum of noise in real time, build a forecast of noise propagation taking into account the meteorological parameters of the situation.

At the same time, there are problems when it is necessary to evaluate and compare acoustic signals in areas remote from each other, which provides for the presence of at least a two-channel measurement system. Such systems can provide an estimate of attenuation of noise during propagation from a source to an operating point in case of non-repeating impulse noises, when assessing the effectiveness of noise protection means, etc. Of particular interest is the use of such tools in the educational process, where, firstly, quite specific tasks of training various specialists can be solved and secondly, the use of highprecision expensive equipment is not always possible.

Thus, the urgent task is to create simple, accessible to students, means of analyzing the noise characteristics of various noise signals based on well-known computer programs using commonly available peripheral devices and systems.

\section{System description}

The program for simultaneous analysis of the spectra of 2 sound signals uses the graphical programming language $\mathrm{G}$ of the LabView environment [5]. The choice of LabView environment is due to the convenience of writing programs and its wide capabilities for creating object-oriented multi-threaded applications for working with devices in real time [5-7].

The block diagram is shown in Fig. 1.

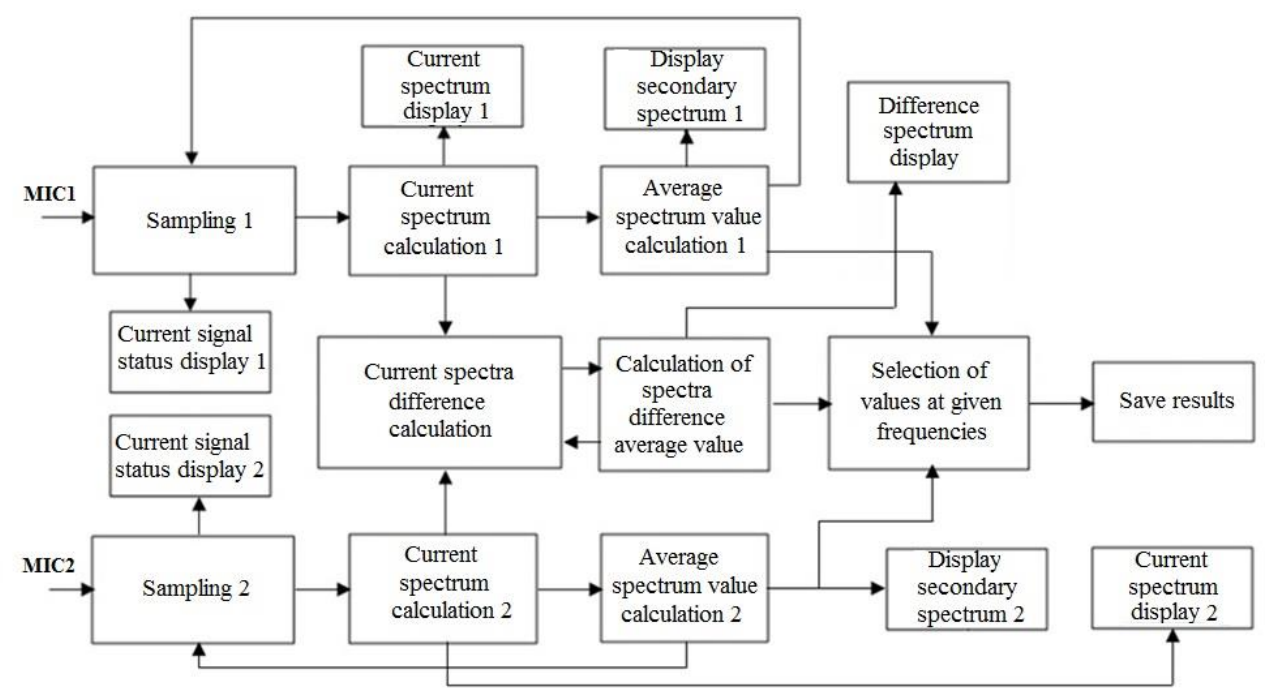

Fig. 1. Block diagram of a two-channel system.

Measurements of the signal from the output of each microphone (MIC1 and MIC2) with the required number of repetitions, determined by the implementation length, are performed in the "Sampling" module of each of the two streams as a separate object. Each object reads the current implementation from 40,000 samples from the output of its microphone and is deleted from the memory after receiving the necessary sequence of implementations. Each measured implementation is sequentially displayed on the chart in real time. Reading of 
individual implementations from the outputs of both microphones takes place in parallel, since the processor is freed up in the pauses between the individual measurements for a time inverse to the sampling frequency.

Each sound signal implementation, obtained in the form of 40,000 16-bit binary numbers, is processed using the FFT in the "Current spectrum calculation" module. The result is the current discrete spectrum of the audio signal in the range from 0 to $20 \mathrm{kHz}$ with a step of $1 \mathrm{~Hz}$, which is also sequentially displayed on a single graph in real time.

The signal spectrum obtained at the next iteration is summed up with the previous, initially zero spectrum, at each discrete frequency. Upon completion of each of the 2 streams (cycles) of measurements, the corresponding spectrum of the sound signal $f_{a v}$ averaged over a given analysis time is calculated, which, after being converted to decibels, is displayed on the screen and stored in a one-dimensional array

$$
f_{a v}(n)=\frac{\sum_{i=1}^{n} x_{i}}{n},
$$

where $x_{i}$ - the received current value of the spectrum, $n$ - the number of measured realizations.

This approach is typical for standard procedures. In this case, another method of obtaining the average value is used. At the end of the averaging cycle, the current average value at each iteration of the averaging cycle is calculated using the inductive function

$$
f_{a v}(n)=\frac{(n-1) \cdot f_{a v}(n-1)+x_{n}}{n} .
$$

The advantage of this option is the ability to observe the change in the average spectrum in real time. In this case, there is no need to set the observation time in advance, since it becomes possible to stop the measurements as soon as the required accuracy is achieved.

The average difference spectrum is calculated in the "Current spectra difference calculation" module as a separate third stream with a given number of repetitions at each iteration. The current spectra, after being converted to decibels, are subtracted at each discrete frequency. The resulting difference is summed with the previous current difference, initially equal to zero. At the end of the third stream, the average spectral difference is calculated, displayed on the screen on a separate graph and stored in a one-dimensional array.

The "Selection of values at given frequencies" module forms a two-dimensional array of the 3 generated one-dimensional arrays containing two average spectra of acoustic signals, as well as the average difference of the logarithmic spectra of the same signals. Each line contains four elements: the value of the selected frequency in hertz, the average values of the spectra of the 1st and 2nd acoustic signals in decibels, as well as the average difference in the logarithmic values (in decibels) of the spectra of the same signals. The generated two-dimensional array is saved in a file in the form of a spreadsheet.

\section{Experimental verification of the system}

The system was verified using a small-sized laboratory acoustic chamber, designed for use in the educational process and research work of students. Its description is given in [8].

The chamber has dimensions of the order of $2 \mathrm{~m}, 1 \mathrm{~m}$ and $0.8 \mathrm{~m}$ with a vertical orientation. On the front side there are two doors providing maximum access to the internal volume. In order to equalize the chamber characteristics and reduce the echo level, the inner surface is covered with acoustic foam. 
An acoustic system built on the basis of broadband dynamic emitters is built into the lower part of the chamber structure [9]. The direction of acoustic signal emission is from the bottom up.

To study the effectiveness of noise screens, guides are provided for installing screening structures at three different levels.

Evaluation of the system performance was carried out using a mock-up of a noise screen having a box design. The internal volume of the screen was filled with sound-absorbing material.

The system used the simplest microphones of the Defender MIC-112 type, one of which was placed in the chamber in front of the screen (in the zone of direct propagation of the acoustic signal), and the second in the same chamber, but after the screen (in the zone of acoustic shadow). The acoustic signal is "white noise" with a fairly uniform distribution within the range of 20-20000 Hz. Assessment time - $60 \mathrm{sec}$.

The measurement results are presented in Fig. 2. The upper spectrum corresponds to the microphone located after the screen (attenuated noise), the middle is to the screen (initial signal), the difference between the spectra is shown in the lower graph. It can be seen that in almost the entire range a decrease in the sound pressure level was recorded, and at a frequency of $1300 \mathrm{~Hz}$ this decrease reaches $50 \mathrm{~dB}$.

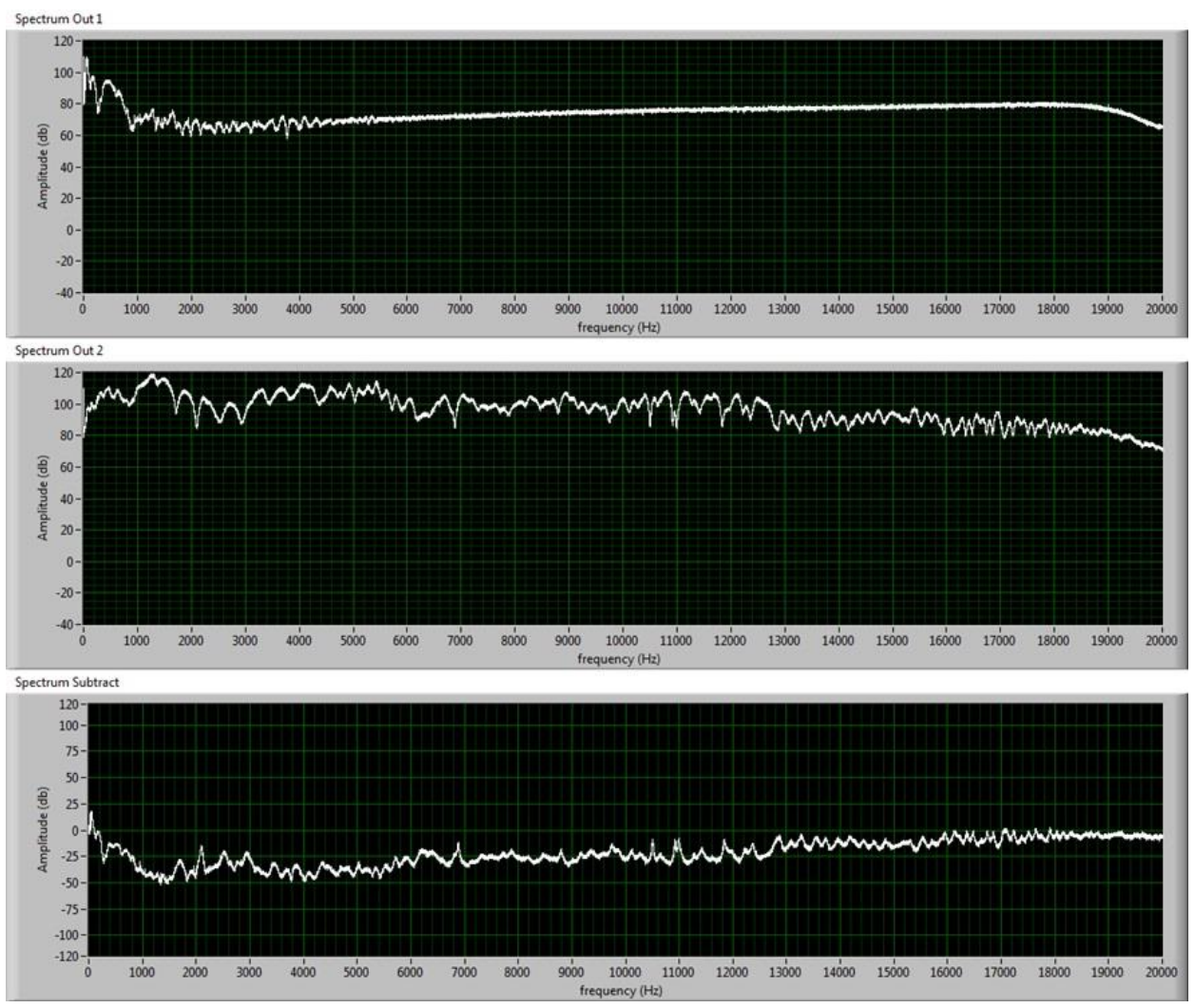

Fig. 2. Measurement results.

\section{Conclusion}

A two-channel system that provides the receipt and processing of control data for diversity of acoustic noise has been developed. The system is implemented in the LabView G 
programming language. Two-channel visual control of changes in the amplitude of the acoustic signal over time, the average spectrum of the signal for each channel, as well as the average difference spectrum of the monitored signals is provided. The possibility of changing the measurement time is obtained.

The advantage of the system is the simplicity of the solution and the ability to measure using widespread means: simple microphones for a personal computer and the PC itself.

The disadvantages include low measurement accuracy, which is determined by the level of the ADC, which is a part of the PC, as well as the microphones used with a large uncorrelated unevenness of AFC.

The system can be used in laboratory or research work in the analysis of spatially separated acoustic noise, the study of means of individual and general protection against noise.

The reported study was funded by RFBR according to the research project No. 18-38-00909.

\section{References}

1. V.V. Bulkin, Noise pollution of industrial cities (the example Murom), Ecological Systems and Devices, v. 1, pp 18-21 (2016)

2. T.D. Shchelokova (Khromulina). The relevance of the study of noise pollution in cities / Monthly International Scientific Journal "Simbol of Science" (Ufa), No. 11-1. - P. 72$74(2015)$

3. IEC 61672-1:2002, Electroacoustics - Sound level meters, Part 1: Specifications (MOD), 31 P. (2012)

4. I.N. Kirillov, V.V. Bulkin, The Mobile System of Urban Area Noise Pollution Monitoring, Problems of Infocom-munications Science and Technology (PIC S\&T'2015), Second International Scientific-Practical Conference. -Kharkov, Ukraine: Kharkiv National University of Radioelectronics, pp. 200-203 (2015)

5. A.Ya. Suranov, LabVIEW 8.20: Feature Reference (DMK Press, 2007)

6. Jeffry Travis, Jim Kring, LabVIEW for Everyone: Graphical Programming MadeEasy and Fun (Crawfordsville: Prentice Hall, 2007)

7. Yu.S. Magda, LabVIEW: A practical course for engineers and developers (DMK Press, 2011)

8. A.A. Badin, A.O. Zavyalov. Laboratory silenced chamber, International scientific journal "Simbol of Science", v. 6, pp.41-43 (2016)

9. A.V. Zaytsev, V.I. Puzyrev, T.S. Sheronova, V.V. Bulkin, Modernization of laboratory acoustic chamber, Methods and devices of information transmission and processing, v. 20, pp. 14-20 (2018) 